\title{
Níveis de ideação suicida em jovens adultos
}

\author{
Levels of suicidal ideation among young adults
}

\author{
José VASCONCELOS-RAPOSO \\ Ana Rita SOARES ${ }^{1}$ \\ Filipa SILVA ${ }^{1}$ \\ Marcos Gimenes FERNANDES ${ }^{2}$ \\ Carla Maria TEIXEIRA ${ }^{1}$
}

\begin{abstract}
Resumo
O suicídio constitui um grave problema para a saúde pública. Está entre as dez principais causas de morte na população mundial em todas as faixas etárias e ocupa o terceiro lugar em jovens entre 15 e 35 anos. O presente estudo é quasi-experimental, quantitativo e transversal, e tem como principal objetivo comparar os níveis de ideação suicida entre estudantes universitários $(n=50)$ versus não universitários $(n=51)$. Os sujeitos foram comparados em função do sexo, idade, local de residência, se vivem acompanhados ou sozinhos, nível de escolaridade e estado ocupacional. O instrumento utilizado foi o Questionário de Ideação Suicida. A amostra foi constituída por 101 indivíduos com idades compreendidas entre 18 e 25 anos, com média de 21,44 anos e desvio-padrão de 2,43. Os resultados evidenciaram que os maiores níveis de ideação suicida encontram-se na população não universitária. Os efeitos estatísticos com significado foram encontrados nas comparações por escolaridade, estatuto ocupacional, viver sozinho e presença de perturbação de ansiedade e/ou depressão. Os resultados do estudo sugerem que sejam adotadas medidas de prevenção do suicídio de forma a reduzir não só a taxa letal, mas também o número de tentativas.
\end{abstract}

Palavras-chave: Adulto jovem; Estudantes; Ideação suicida.

\begin{abstract}
Suicide is a serious public health problem and is among the top ten causes of death worldwide for all age groups. It is the third leading cause among 15-35-year olds. The main objective of this quasi-experimental, quantitative, and cross-sectional study was to compare the levels of suicidal ideation among college students $(n=50)$ and nonstudents $(n=51)$. Subjects were compared according to sex, age, place of residence, living arrangements, educational level, and occupational status using the Suicidal Ideation Questionnaire. The sample consisted of 101 individuals aged 1825 years, Mean of 21.44 years and Standard Deviation of 2.43. The results showed higher levels of suicidal ideation among the non-college students. Statistically significant effects were found among the following variables: level of education, occupational status, living alone, and presence of anxiety disorder and/or depression. The findings of this study suggest the need for suicide prevention measures in order to reduce deaths by suicide and suicide attempt rate.
\end{abstract}

Keywords: Young adult; Students; Suicide ideation.

\footnotetext{
$\nabla \nabla \nabla v$
}

1 Universidade de Trás-os-Montes e Alto Douro, Departamento de Educação e Psicologia, Instituto de Engenharia de Sistemas e Computadores - Tecnologia e Ciência. R. Dr. Manuel Cardona, Apartado 1013, 5000-801, Vila Real, Portugal. Correspondência para/ Correspondence to: J. VASCONCELOS-RAPOSO.E-mail: <jvraposo@utad.pt>.

2 Universidade Estadual de Santa Cruz, Centro de Ciências Biológicas, Departamento de Ciências da Saúde. Ilhéus, BA, Brasil. 
De acordo com a Organização Mundial da Saúde, anualmente, cerca de um milhão de pessoas cometem suicídio e espera-se que esse número suba para um milhão e quinhentos mil (Organização Mundial da Saúde, 2013). Segundo Figel, Menegatti e Pinheiro (2013) esse valor representa cerca de $15 \%$ das mortes que acontecem em nível mundial. É estimado que para cada suicídio ocorrido haja cerca de 20 tentativas. Em face a esses valores, é da maior importância que se realizem estudos que permitam um conhecimento mais aprofundado sobre as variáveis que podem influenciar os comportamentos suicidas, de forma a tornar possível a intervenção preventiva em múltiplos contextos.

Na perspectiva dos estudos aplicados, a pesquisa tem centrado a sua análise nos relatos de sujeitos que tentaram o suicídio ou, ainda, tentado entender como as diversas contingências da vida dos indivíduos podem ter servido como facilitadores do ato, uma vez que há uma correlação positiva entre ideação suicida e sua concretização (Figel et al., 2013; Prieto \& Tavares, 2005). O entendimento sobre a forma como essas contingências podem contribuir para o suicídio poderá proporcionar mais conhecimentos para o planejamento e implementação de estratégias que visem a prevenção.

No presente estudo, de forma a dar um contributo efetivo para essa área do saber, aborda-se a ideação suicida a partir de seus dois polos: a tentativa ou o suicídio efetivo. O propósito geral é identificar e diferenciar sujeitos em função das ideias, desejos ou pensamentos que possam ter eventualmente quanto a morrer de forma intencional.

O comportamento suicida tem sido conceitualizado como um contínuo de pensamentos e comportamentos desde a ideação até o suicídio em si (Ganz, Braquehais, \& Sher, 2010). Segundo a Organização Mundial da Saúde (2006), o suicídio encontra-se entre as dez principais causas de morte em todo o mundo, sendo estimado que aproximadamente 10 a 20 milhões de pessoas o contemplem pelo menos uma vez na vida. Além disso, ao longo dos últimos 50 anos constatou-se um aumento de $60 \%$ no número de suicídios em escala mundial, representando a terceira causa de morte em jovens entre 15 e 35 anos de idade (Gonçalves, Freitas, \&
Sequeira, 2011). Cerca de um milhão de indivíduos suicidaram-se durante o ano 2000 e as estimativas sugerem que há uma tendência para o aumento do número de tentativas (Van Orden et al., 2010). Como exemplo desse fato, o estudo de Pereira (2011), realizado numa população universitária, concluiu que metade dos $12 \%$ dos alunos os quais declararam já ter tido ideação suicida chegaram a sentir-se determinados a cometer o suicídio.

Um estudo realizado na Hungria oferece uma noção mais específica de fatores que podem estar envolvidos no risco associado às ideias, tentativas e concretizações suicidas. Com efeito, Almasi et al. (2009) sugerem que os maiores fatores de risco tendem a estar associados a fatores sociodemográficos, nomeadamente o desemprego, a baixa escolaridade, o estado civil, bem como o isolamento social (Moreira \& Gonçalves, 2010). Apresentam-se, ainda, como fatores de risco acrescentado, a prevalência de fatores clínicos, como a existência de diagnósticos prévios de doença mental, com particular destaque para a depressão e a esquizofrenia, assim como os casos de abuso de álcool e drogas. Como condições protetoras, o estudo identificou a prática religiosa e o suporte social.

Os fatores de proteção incidem em quatro esferas de indicadores, nomeadamente os estilos cognitivos e as características de personalidade, o modelo familiar, os fatores culturais, sociais e as crenças religiosas (Organização Mundial da Saúde, 2006), bem como os ambientais (Gonçalves et al., 2011). Por outro lado, a literatura aponta como fatores de risco mais consistentes e indicadores de suicídio os sujeitos apresentarem uma história de tentativas anteriores, isolamento social, conflitos familiares, desemprego e doenças físicas (Van Orden et al., 2010). Outro indicador consistente referente à epidemiologia do comportamento suicida é a diferença de gêneros, pois os índices de suicídio masculino superam o feminino em todo o mundo. No entanto, a maior porcentagem de tentativas está entre as mulheres (Cash \& Bridge, 2009; Eaton et al., 2008; Van Orden et al., 2010). Quanto às práticas utilizadas, os homens tendem a recorrer a métodos mais violentos.

As associações entre o desemprego e as taxas de suicídio têm sido alvo de diversas inves- 
tigações ao longo dos anos. Lewis e Sloggett (1998) reportam um estudo do Office for National Statistics que associa as taxas de suicídio, o desemprego, o estado socioeconômico e a doença crônica. A pesquisa, realizada entre 1971 e 1993, apresentou resultados com uma elevada associação entre o desemprego e as taxas de suicídio, com maior incidência no sexo masculino. Essa relação já havia sido evidenciada no estudo de Crombie (1989).

Mudanças socioeconômicas bruscas também estão, geralmente, associadas ao suicídio (Denney, Rogers, Krueger, \& Wadsworth, 2009). Sobre esse fato, foi desenvolvido um estudo na Coreia do Sul entre 1993 e 2006 (Lee et al., 2009) com o objetivo de identificar fatores que ajudassem a compreender essa relação. A pesquisa contemplou o gênero, a idade, o local de residência e os níveis de escolaridade como variáveis independentes e concluiu que as taxas de suicídio estão mais concentradas nos grupos com baixa escolaridade, o que se verificou em ambos os gêneros.

No que diz respeito aos estudos os quais consideram o estado civil em associação com a taxa de suicídio, parece existir consistência nos resultados, sendo que essa taxa diminui quando o estado civil corresponde a casado(a). O mesmo não acontece quando se trata de outros estados, nomeadamente divorciado(a), solteiro(a) ou viúvo(a), já que estes evidenciam taxas mais elevadas (Denney et al., 2009).

Émile Durkheim, em sua obra "Suicídio" (Durkheim, 1952), argumentou que a religião desempenha um papel relevante frente às alterações nas taxas desse tipo de prática. Torna-se importante, assim, estudar esse fenômeno. Com esse propósito, foi desenvolvida a escala Reasons for Living, um instrumento de avaliação que contempla razões impeditivas do suicídio e da qual fazem parte questões relativas à religiosidade. No caso da tradição Judaico-Cristã a prática do suicídio é repudiada e rejeitada. A teoria sugere que, devido aos princípios enumerados nas Escrituras Sagradas, a ideia de violar a religião inibe o indivíduo quanto a praticar o ato ou mesmo refletir sobre a hipótese. Já no que diz respeito às religiões politeístas, o suicídio, apesar de não ser apoiado, também não é condenado ou punido, pois, segundo essas crenças, o homem tem direito a pôr termo em sua vida, uma vez que se prevê que irá reencarnar após a sua morte. No entanto, as religiões politeístas também fomentam o respeito pela vida humana. Nesse quadro, as práticas religiosas, mais concretamente as monoteístas, têm uma influência positiva na diminuição das taxas de suicídio (Bteshe, Oliveira, Clébicar, Estellita-Lins, \& Salles, 2010).

O início da vida universitária leva à ocorrência de múltiplas mudanças na dinâmica dos jovens estudantes. Esse período, marcado por desafios e incertezas, pode dar origem a vários problemas de saúde mental, entre eles o comportamento suicida (Gonçalves et al., 2011). A ideação suicida entre estudantes universitários pode ter uma etiologia específica devido à presença de pressões sociais e acadêmicas que distinguem essa população (Wilcox et al., 2010). A presença de maiores níveis de apoio social parece exercer um efeito protetor contra esse tipo de comportamento (Arria et al., 2009); por outro lado, o isolamento e a falta de suporte social apresentam-se como elementos facilitadores (Arria et al., 2009; Joiner, 2005; Joiner et al., 2009).

Os fatores clínicos também assumem variáveis importantes no que diz respeito à análise do comportamento suicida. Dentro dos diversos quadros clínicos destacam-se a ansiedade e a depressão, quer seja de forma independente ou em comorbidade. Esses transtornos estabelecem uma elevada associação com a ideação suicida (Denney et al., 2009; Galbán, Rodriguez, Cruz, Arencibia, \& Álvarez, 2002; Lee et al., 2009; Nock et al., 2009; Rivera, 2010). As perturbações caracterizadas pela presença de ansiedade emergem como fortes e úteis preditoras de ideação e de tentativas não planeadas (Joe, Baser, Neighbors, Caldwell, \& Jackson, 2009; Kessler, Borges, \& Walters, 1999; Nock et al., 2009). Estima-se que cerca de $90 \%$ dos indivíduos que cometeram suicídio tenham tido alguma perturbação de foro mental e que, no momento do ato, $60 \%$ estavam deprimidos (Organização Mundial da Saúde, 2006).

Vários estudos (Galbán et al., 2002; Rivera, 2010) sugerem que a idade crítica para a ideação e tentativas suicidas, em pacientes com elevados 
níveis de ansiedade, insônias e sintomas depressivos, está entre os 17 e os 23 anos. Por outro lado, e contrariando esse fato, ainda que a presença de depressão fosse um fator de risco para a ideação, Arria et al. (2009) afirmaram que a maioria dos indivíduos da pesquisa que apresentaram ideação suicida (cerca de 60\%) não satisfazia o critério para altos níveis de sintomatologia depressiva.

Para além dos aspectos até agora referidos na literatura, deve-se levar em consideração o fato de Portugal viver, desde 2008, uma crise financeira com repercussões socioemocionais acentuadas na população. Os elevados níveis de desemprego, $35,6 \%$ em jovens com idade entre 15 e 24 anos (Instituto Nacional de Estatística, 2013), fazem questionar sobre qual o impacto dessa situação nos estados emocionais desses cidadãos. Se nos últimos 20 anos ser detentor de um grau acadêmico era um fator importante para se ter acesso a boas condições de vida, agora, obter um diploma pouco significa em termos de futuro. No entanto, para os universitários existem maiores possibilidades de encontrar trabalho no mercado de emprego europeu, caso optem por emigrar. Porém, é previsível que os indivíduos os quais não tiveram a oportunidade de estar melhor classificados para a nova realidade do mercado de trabalho, enfrentam maiores dificuldades em conceber o futuro de forma positiva.

Assim, o objetivo geral deste estudo consiste em comparar estudantes universitários e indivíduos não universitários quanto à incidência de ideação suicida. Os objetivos específicos consistem em comparar jovens por sexo, idade, deslocamento (ou não) da residência familiar, se vivem sozinhos, nível de escolaridade, estatuto social, estado civil, grau de religiosidade e se alguma vez foram (ou não) diagnosticados com doença mental.

\section{Método}

O estudo é quasi-experimental, quantitativo e transversal, e tem como principal objetivo comparar os níveis de ideação suicida entre estudantes universitários versus indivíduos não universitários, atualmente residentes no interior do norte de Portu- gal. A amostra foi constituída por 101 pessoas, sem história de tratamento psiquiátrico e com idades compreendidas entre os 18 e os 25 anos, com média de 21,44 anos e desvio-padrão de 2,43. Após obtenção de concordância em participar do estudo, todos os sujeitos foram informados que poderiam abandoná-lo a qualquer momento.

A maior parte dos elementos da amostra foi do sexo feminino $(n=59)$, solteiros $(n=97)$, que vivem deslocados da sua residência habitual $(n=79)$ e que, na cidade onde se encontram, vivem sozinhos $(n=88)$. Dos que não moram sozinhos, 45 vivem com amigos, 36 com a família e os restantes $(n=21)$ com outras pessoas. Quanto ao grau de escolaridade, 50 sujeitos frequentam o ensino superior, 37 possuem o ensino secundário, $13,03^{\circ}$ ciclo completo e apenas 1 possui até o $2^{\circ}$ ciclo. Por recomendação do Comité de Ética, a análise por área científica não foi considerada. De qualquer forma, houve a preocupação em manter o mais semelhante possível a porcentagem de estudantes representantes das áreas científicas existentes nas instituições de ensino superior de onde se retirou a amostra.

Relativamente ao estatuto ocupacional, predominam os estudantes $(n=50)$ seguidos dos empregados $(n=30)$ e dos desempregados $(n=21)$. Quando divididos por estado civil, 24 são casados e 77 solteiros. Os indivíduos que integram a amostra são majoritariamente religiosos (62\%), sendo que todos foram questionados a respeito do quão religiosos se consideravam. As resposta foram obtidas numa escala de 0 (nada religiosos) a 10 (muito religiosos). Quando questionados sobre se alguma vez Ihes foi diagnosticada uma perturbação de ansiedade ou depressão, 83 sujeitos responderam "não" e 18, "sim", sendo que, destes, 8 referiram ter sido ansiedade e 10, depressão.

\section{Instrumentos}

O grau de ideação suicida foi avaliado pelo Questionário de Ideação Suicida (QIS), versão portuguesa do Suicide Ideation Questionnaire, de Reynolds (1988), traduzida e adaptada para a população portuguesa por Ferreira e Castela (1999). O QIS tem como objetivo analisar a ocorrência de ideação 
suicida em adolescentes e adultos. É constituído por 30 itens (e.g., Pensei que suicidar-me resolveria os meus problemas) com respostas estruturadas numa escala de Likert com 7 valores: 0 - nunca tive este pensamento; 1 - pensei nisto, mas não no último mês; 2 - cerca de uma vez por mês; 3 - duas vezes por mês; 4 - cerca de uma vez por semana; 5 - duas vezes por semana; 6 - quase todos os dias. O valor da escala é obtido somando-se as pontuações dos 30 itens, e varia entre 0 e 180 pontos. Quanto maior a pontuação, maior a ideação suicida.

No que diz respeito à consistência interna, no estudo de validação do QIS para a população portuguesa (Ferreira \& Castela, 1999) foi obtido um valor do Alfa de Cronbach de 0,96. No presente estudo, o valor do Alfa de Cronbach foi de 0,978, o que indica uma boa consistência interna da escala do QIS.

\section{Procedimentos}

Os dados do estudo relativos à população estudantil foram recolhidos em instituições de ensino superior universitário no norte de Portugal com a devida avaliação e autorização das entidades acadêmicas. Com relação à população não estudante, os questionários foram distribuídos pelos pesquisadores aos indivíduos que cumprissem os critérios de inclusão, ou seja, serem residentes na mesma área dos universitários.

As variáveis independentes deste estudo foram a idade, o sexo, deslocamento do local de residência habitual, com quem vive atualmente, o nível de escolaridade, o estado civil, a ocupação, a religião e o diagnóstico de depressão ou de ansiedade. A variável dependente foi a ideação suicida. O projeto foi aprovado pelo Comitê de Ética (Processo n 32r/2012) e, consequentemente, autorizado pela administração das instituições onde as informações foram recolhidas.

O tratamento estatístico dos dados foi realizado através do pacote estatístico Statistical Package for the Social Sciences (SPSS, versão 21). Para a análise estatística, considerou-se o valor de $p \leq 0,05$ para rejeitar a hipótese nula. A normalidade dos dados foi definida através da observação do histograma, bem como dos valores dos coeficientes de assimetria $(1,958)$ e curtose $(3,360)$. Constatou-se que a variável dependente em estudo (de ideação suicida) não apresentou uma distribuição normal. Desta forma, optou-se pela realização de testes não paramétricos: Teste de Mann-Whitney, para fazer as comparações entre 2 grupos, e Teste de Kruskall-Wallis, quando se compararam 3 ou mais grupos (Marôco, 2010).

\section{Resultados}

À seguir são apresentados os resultados relativos às comparações feitas por sexo, idade, local de residência, se os sujeitos vivem sozinhos ou acompanhados, nível de escolaridade, estatuto ocupacional, estado civil, religiosidade e a existência ou não de diagnóstico prévio de doença mental.

\section{Níveis de ideação suicida}

Os níveis de ideação suicida da amostra variaram entre 0 e 119, com média de 20,15 e desvio-padrão de 28,11. Os dados evidenciaram que a maioria dos indivíduos apresentou baixos níveis de ideação suicida, verificando-se que cerca de metade obteve valores inferiores a 8 , e que $25 \%$ apresentou valores superiores a 26,5 .

De acordo com Reynolds (1988), uma pontuação no QIS superior ou igual a 41 pontos pode ser indicativa da presença de psicopatologia e de um potencial risco de suicídio. Na amostra, verificou-se que existem 16 indivíduos com pontuação superior ou igual a 41. Os resultados evidenciaram que 8 dos sujeitos em risco estavam desempregados, 6 eram estudantes e 2 eram não-estudantes e empregados.

\section{Comparação dos níveis de ideação}

No que diz respeito às comparações por sexo, recorreu-se ao Teste de Mann-Whitney, o qual demonstrou não existirem diferenças entre os valores medianos dos dois sexos $(U=1052,50 ; W=2822,50$; 
$p=0,198)$. Já para verificar se a ideação suicida varia com a idade, foram considerados 4 escalões etários e utilizou-se o teste de Kruskall-Wallis. Os resultados do teste $\left(\chi^{2}=5,689 ; p=0,128\right)$ sugerem a não existência de diferenças estatisticamente significativas entre os quatro escalões etários considerados (18-19; 20-21; 22-23; 24-25). O coeficiente de correlação de Spearman obtido $\left(R_{s D}=0,146\right.$; $\left.p=0,146 ; r^{2}=0,020\right)$ indicou uma correlação positiva mas não significativa entre a idade e a ideação suicida.

Quanto à deslocalização do local habitual de residência, também não foram verificadas diferenças estatisticamente significativas entre deslocados e não-deslocados $(U=710,50 ; W=3870,50$; $p=0,191)$. Todavia, foram constatadas diferenças quando as comparações foram feitas em função do viverem sozinhos ou com outra pessoa, através do Teste de Mann-Whitney $(U=363,00 ; W=4279,00$; $p=0,034)$. Verificou-se que os indivíduos os quais moram sozinhos apresentaram níveis de ideação suicida superiores ao que vivem acompanhados. No entanto, quando se questionou sobre se os indivíduos vivem com familiares ou não, o Teste de Kruskall-Wallis $\left(\gamma^{2}=2,136 ; p=0,344\right)$ demonstrou não existirem diferenças entre os que vivem com a família, com os amigos ou com outras pessoas.

Para comparar os níveis de ideação suicida por escolaridade, agruparam-se os sujeitos com o $2^{\circ}$ e o $3^{\circ}$ ciclo. Os resultados demonstraram que as habilitações literárias influenciam de forma importante os níveis de ideação suicida $\left(\gamma^{2}=8,453\right.$; $p=0,015)$. Os testes da comparação múltipla evidenciaram diferenças estatisticamente significativas entre os indivíduos que frequentavam o ensino universitário e os que tinham apenas o $2^{\circ}$ ou $3^{\circ}$ ciclos $(t=25,713 ; p=0,004)$, e entre os que tinham o ensino secundário e os que tinham o $2^{\circ}$ ou $3^{\circ}$ ciclos ( $t=20,237 ; p=0,027$ ). Os resultados demonstraram que quanto menor o grau de escolaridade maior a ideação suicida.

Relativamente ao estatuto ocupacional, constatou-se uma influência da variável nos níveis de ideação suicida $\left(\gamma^{2}=10,226 ; p=0,006\right)$. Os testes da comparação múltipla indicaram diferenças e os desempregados ( $t=-23,641 ; p=0,002)$ e entre os empregados e os desempregados $(t=-21,438$; $p=0,010)$. Os desempregados apresentaram valores de ideação suicida superiores aos empregados e aos estudantes.

Quanto ao estado civil, não existiram diferenças dos valores medianos do QIS entre solteiros e casados $(U=179,50 ; W=4932,50 ; p=0,800)$. $O$ mesmo se verificou no que diz respeito à religião, onde também não foram identificadas diferenças dos níveis de ideação suicida entre os religiosos e os não religiosos ( $U=1061,50 ; W=3014,50 ; p=0,303)$. Foi também estudada a correlação entre os valores do QIS e a variável que definia, numa escala de 0 a 10 , a medida relativa a quanto os sujeitos se consideravam religiosos. O coeficiente de correlação de Spearman obtido $\left(R_{S p}=-0,171 ; p=0,088 ; r^{2}=0,03\right)$ indicou uma correlação negativa baixa e não significativa entre as duas variáveis.

Por último, quanto à existência prévia de um diagnóstico de doença mental, os resultados mostraram que os indivíduos diagnosticados previamente com uma perturbação mental (ansiedade ou depressão) apresentaram níveis de ideação suicida superiores aos outros, sendo as diferenças estatisticamente significativas $(U=319,50 ; W=3805,50$; $p=0,001)$. No entanto, quanto ao tipo de perturbação diagnosticada, não se verificaram diferenças entre os sujeitos com depressão e os com ansiedade $(U=29,50 ; W=65,50 ; p=0,350)$.

\section{Discussão}

O presente trabalho visou comparar estudantes universitários com outros jovens em função do sexo, idade, proximidade residencial com a família, se vivem sozinhos ou acompanhados, grau de escolaridade, se trabalham ou não, estado civil, nível de religiosidade e se alguma vez lhes foi diagnosticada uma doença mental. No que concerne às diferenças na taxa de ideação suicida entre estudantes universitários e indivíduos não-universitários, verificou-se que existe uma baixa porcentagem de pessoas com valores significativos de ideação suicida. No entanto, entre aqueles que apresentam essa característica predominam os jovens 
adultos não-universitários. Assim, a variável "estudante universitário" não apresentou valores críticos no domínio de ideação suicida. Quando comparados universitários com não-universitários, os dados sugerem que a vida universitária, por si só, não se constitui como elemento facilitador da ideação suicida. Os resultados desta pesquisa corroboram os de Pereira (2011). Assim, estudos que abordem o problema de suicídio entre universitários deverão incluir outras variáveis para que haja um maior rigor da análise da taxa de ideação suicida entre eles.

Relativamente às comparações por sexo, não foram encontradas diferenças. Esses dados contrariam os encontrados em outros estudos os quais sugerem que é entre os homens que se observam mais comportamentos suicidas (Eaton et al., 2008; Van Orden et al., 2010). A existência de diferenças entre universitários e não-universitários, com relação à ideação suicida, poderá ser um indicador de que o suicídio no grupo de estudantes poderá ter uma etiologia específica, devido à presença de pressões sociais e acadêmicas que distinguem essa população das restantes, enquanto entre os não-universitários verificou-se resultados consistentes com os da literatura desde há muito evidenciados (Durkheim, 1952).

Existem várias características relativas a esse período de transição do desenvolvimento, nomeadamente o fato dos sujeitos abandonarem provisoriamente a casa dos pais, a criação ou não de redes de apoio entre pares, bem como a experiência de ambientes não familiares, as quais contribuem para algum tipo de vulnerabilidade na vida universitária dos jovens. Essas características, por sua vez, poderão facilitar um acréscimo na predisposição destes para experienciar problemas de saúde mental (Wilcox et al., 2010).

Um dos aspectos que, eventualmente, poderia contribuir para o aumento dessa vulnerabilidade pode ser o afastamento do seu local de residência habitual, mas, na presente amostra, não foram encontradas quaisquer diferenças entre os indivíduos deslocados e os não deslocados. A explicação para esses dados pode residir no fato de que os jovens, quando ingressam na universidade, tendem a estar sujeitos a um conjunto de práticas (praxe) que, de alguma forma, podem contribuir para contrariar os efeitos nefastos que resultam do isolamento social, uma vez que da interação intensa mantida ao longo de, aproximadamente, um mês emergem laços de amizade e de companheirismo que num número elevado de sujeitos se traduz em amizades para o resto da vida. Em outras palavras, a intensificação da vida social nessa primeira fase de adaptação à nova vida, enquanto estudantes, pode possibilitar a implementação de mecanismos que evitam o isolamento, e isso, por sua vez, poderá, eventualmente, atenuar a ocorrência de ideação suicida. De qualquer forma, esse é mais um dos aspectos que reforçam a ideia de uma etiologia específica para estudantes universitários.

Contudo, no que refere à questão "Com quem vive?", verificou-se que os resultados são concordantes com os de outros autores (Arria et al., 2009; Joiner, 2005; Joiner et al., 2009), ou seja, existem, de fato, diferenças significativas, com maior índice de ideação suicida naqueles indivíduos que vivem sozinhos. Assim, o isolamento e a falta de suporte social apresentam-se como uma influência na ocorrência desse tipo de comportamento.

No que concerne à ideação suicida, entre os fatores que são descritos como sendo de risco, encontra-se, entre outros, o baixo nível de escolaridade. O presente estudo corrobora resultados os quais sugerem que quanto maior o grau de escolaridade menor o valor de ideação suicida (Almasi et al., 2009; Denney et al., 2009; Lee et al., 2009). Algumas das explicações avançadas para essa ocorrência prendem-se ao argumento de que os indivíduos com baixos níveis de escolaridade tendem a ter maiores dificuldades em conseguir um trabalho melhor, com melhores níveis de remuneração, e que Ihes possibilite evoluir pessoal e profissionalmente. Há, ainda, os que teorizam que a uma baixa escolaridade estão associados os empregos com menor estabilidade financeira e sentimento de insegurança pessoal e familiar. Em contrapartida, pessoas com um nível de escolaridade mais elevado têm acesso a um maior conhecimento de medidas preventivas e de fatores de risco associados às mais diversas questões com que se deparam nas suas vida quotidianas. Em acréscimo aos argumentos já referidos, há evidências de que a baixos índices de escola- 
ridade está associada uma maior propensão para o desenvolvimento de desordens de foro psicológico. No seu conjunto, os aspectos referidos exercem um efeito agregador dos fatores de risco frente ao suicídio (Denney et al., 2009; Lee et al., 2009).

Quanto ao estatuto ocupacional dos indivíduos, os resultados do presente estudo corroboram aqueles de pesquisas previamente realizadas (Almasi et al., 2009; Lewis \& Sloggett, 1998; Van Orden et al., 2010), as quais identificaram o nesse sentido, o estar empregado pode funcionar como um fator preventivo. Ter um emprego mostra-se relevante para além do fator econômico, pois acarreta, também, uma dimensão de integração social, oferecendo oportunidades para organizar o tempo quotidiano de forma rentável, fazer amigos e ajudando a ultrapassar, mais facilmente, situações difíceis com comportamentos culturalmente ajustados (Denney et al., 2009).

No que diz respeito ao estado civil, demonstrou-se que não existem diferenças significativas entre indivíduos casados e solteiros. Isso contraria a sugestão do estudo de Denney et al. (2009), onde argumenta-se que exista uma maior probabilidade de uma taxa mais elevada de suicídio em indivíduos solteiros. No caso do presente estudo a amostra é mais jovem, o que pode, de alguma forma, explicar as diferenças encontradas em ambas as pesquisas. Acrescenta-se, ainda, a tendência que atualmente se constata na sociedade portuguesa: as pessoas tendem a casar e constituir família em uma idade mais avançada.

A religião tende a ser apresentada na literatura como um fator protetor frente ao suicídio, sendo que, nas monoteístas, o suicídio é tido como um ato de punição e desrespeito aos princípios da própria religião (Bteshe et al., 2010). No entanto, no presente estudo, não foram encontradas diferenças significativas em função da religiosidade. Em parte, esse fato pode advir do efeito combinado do suporte social vivenciado em contexto universitário, assim como da perda de importância da religião como fonte de critérios normativos para a vida das pessoas e dos jovens de uma forma particular.

Os fatores clínicos, principalmente o fato de 352 já terem sido diagnosticados com depressão e/ou ansiedade, evidenciaram-se, em investigações anteriores, como preditores de ideação suicida (Denney et al., 2009; Galbán et al., 2002; Lee et al., 2009; Nock et al., 2009; Rivera, 2010). No atual estudo, constatou-se que a taxa de ideação foi superior em indivíduos que apresentaram uma dessas perturbações mentais no passado. No entanto, quando comparados os sujeitos em função do diagnóstico de depressão ou de ansiedade, não foram encontradas diferenças significativas. Eventualmente, esses resultados podem ser explicados pela reconhecida comorbidade entre essas patologias. Outro aspecto o qual deve ser levado em consideração é que não foi avaliada a severidade dos sintomas.

Apesar de o suicídio corresponder à terceira maior causa de morte em jovens com idades compreendidas entre 15 e 35 anos (Gonçalves et al., 2011), no presente estudo constatou-se algumas diferenças na taxa de ideação suicida nas comparações relativas às variáveis independentes "viverem sozinhos ou acompanhados", "grau de escolaridade" e "estatuto ocupacional". As diferenças encontradas nem sempre corroboraram os resultados de estudos realizados anteriormente.

Os resultados obtidos nesta investigação permitem concluir que estar desempregado se constitui como um acréscimo em termos de risco para a ideação suicida. Viver sozinho também está associado a maiores índices de ideação suicida. Tal como verificado em outros estudos, também na presente amostra o nível educacional mais baixo tendeu a estar associado a uma maior ideação suicida, assim como o fato de já ter sido diagnosticado com patologia mental. Recomenda-se, entretanto, que em estudos futuros leve-se em consideração a severidade dos sintomas. Os resultados sugerem que a degradação das condições de vida e a falta de oportunidades de emprego sentidas pela população jovem de Portugal constituem fatores de risco para a ideação suicida.

\section{Colaboradores}

J. VASCONCELOS-RAPOSO foi co-responsável pela concepção e pelo tratamento de dados de supervisão 
científica do projeto. A. R. SOARES e F. SILVA procederam a coleta e construção da base de dados sendo co-autoras na construção do $1^{\circ}$ draft do estudo. M. G. FERNANDES participou na revisão do texto e acompanhou os processos burocráticos do projeto. C. M. TEIXEIRA foi responsável pela supervisão da escrita, da revisão do texto, da coerência teórica e metodológica do projeto.

\section{Referências}

Almasi, K., Belso, N., Kapur, N., Webb, R., Cooper, J., Hadley, S., ... Appleby, L. (2009). Risk factors for suicide in Hungary: A case-control study. Biomed Central Psychiatry, 9(45), 1-9. http://dx.doi.org/10.1186/1471-2 44X-9-45

Arria, A., O'Grady, K., Caldeira, K., Vincent, K., Wilcox, H., \& Wish, E. (2009). Suicide ideation among college students: A multivariate analysis. Archives of Suicide Research, 13(3), 230-246. http://dx.doi.org/10.1080/ 13811110903044351

Bteshe, M., Oliveira, V., Clébicar, T., Estellita-Lins, C., \& Salles, I. (2010). Suicídio na literatura religiosa: o kardecismo como fonte bibliográfica privilegiada. Revista Electrónica de Comunicação, Informação e Inovação em Saúde, 4(3), 37-50. http://dx.doi.org/10.3395/ reciis.v4i3.384pt

Cash, S., \& Bridge, J. (2009). Epidemiology of youth suicide and suicidal behavior. Current Opinion in Pediatrics, 21(5), 613-619. http://dx.doi.org/10.1097/ MOP.0b013e32833063e1

Crombie, I. (1989). Trends in suicide and unemployment in Scotland, 1976-86. British Medical Journal, 298(6676), 782-784.

Denney, J., Rogers, R., Krueger, P., \& Wadsworth, T. (2009). Adult suicide mortality in the United States: Marital status, family size, socioeconomic status and differences by sex. Social Science Quarterly, 90(5), 1167-1185. http://dx.doi.org/10.1111/j.1540-6237. 2009.00652.x

Durkheim, E. (1952). Suicide: A study in Sociology. London: Free Press.

Eaton, D., Kann, L., Kinchen, S., Shanklin, S., Ross, J., Hawkins, J., ... Wechsler, H. (2008). Youth risk behavior surveillance - United States, 2007. Morbidity and Mortality Weekly Report Surveillance Summaries, 57(5504), 1-131.

Ferreira, J., \& Castela, M. (1999). Questionário de Ideação Suicida (Q.I.S). In M. R. Simões, M. M. Gonçalves, \& L. S. Almeida (Eds.), Testes e provas psicológicas em Portugal (pp.129-130). Braga: Sistemas Humanos e Organizacionais.

Figel, F., Menegatti, C., \& Pinheiro, E. (2013). Tentativas de suicídio: uma análise de contigências. Estudos de
Psicologia (Campinas), 30(2), 211-218. http://dx.doi. org/10.1590.S0103-166X2013000200007

Galbán, Y., Rodríguez, L., Cruz, M., Arencibia, T., \& Álvarez, M. (2002). Comportamiento del intento suicida en un grupo de adolescentes y jóvenes. Revista Cubana Medicina Militar, 31(3), 182-187.

Ganz, D., Braquehais, M. D., \& Sher, L. (2010). Secondary prevention of suicide. PLoS Medicine, 7(6), 1-4. http:// dx.doi.org/10.1371/journal.pmed.1000271

Gonçalves, A., Freitas, P., \& Sequeira, C. (2011). Comportamentos suicidários em estudantes do ensino superior: factores de risco e de proteção. Millenium, 40, 149159.

Instituto Nacional de Estatística. (2013). Estatísticas do emprego - 2013: $3^{\circ}$ trimester. Lisboa: Autor.

Joe, S., Baser, R., Neighbors, H., Caldwell, H., \& Jackson, J. (2009). 12-Month and lifetime prevalence of suicide attempts among black adolescents in the national survey of american life. Journal American Academy Child Adolescent Psychiatry, 48(3), 271-282. http:// dx.doi.org/10.1097/CHI.0b013e318195bccf

Joiner, T. (2005). Why people die by suicide. Cambridge: Harvard University Press.

Joiner, T., Van Orden, K., Witte, T., Selby, E., Ribeiro, J., Lewis, R., \& Rudd, D. (2009). Main predictions of the interpersonal-psychological theory of suicidal behavior: Empirical tests in two samples of young adults. Journal of Abnormal Psychology, 118(3), 634-646. http://dx. doi.org/10.1037/a0016500

Kessler, R., Borges, G., \& Walters, E. (1999). Prevalence of and risk factors for lifetime suicide attempts in the National Comorbidity Survey. Archives of General Psychiatry, 56(7), 617-626.

Lee, W., Khang, Y., Noh, M., Ryu, J., Son, M., \& Hong, Y. (2009). Trends in educational differentials in suicide mortality between 1993-2006 in Korea. Yonsei Medical Journal, 50(4), 482-492. http://dx.doi.org/ 10.3349/ymj.2009.50.4.482

Lewis, G., \& Sloggett, A. (1998). Suicide, deprivation and unemployment: Record linkage study. British Medical Journal, 317(7168), 1283-1286.

Marôco, J. (2010). Análise estatística com o PASW Statistics (ex-SPSS). São Paulo: ReportNumber.

Moreira, N., \& Gonçalves, R. (2010). Perturbação mental e ideação suicida entre reclusos preventivos. Análise Psicológica, 1(28), 133-148.

Nock, M., Hwang, I., Sampson, N., Kessler, R., Angermeyer, M., Beautrais, A., ... Williams, D. (2009). Cross-national analysis of the associations among mental disorders and suicidal behavior: Findings from the WHO World Mental Health Surveys. PLOS Medicine, 6(8), 1-17. http://dx.doi.org/10.1371/ journal.pmed.1000123

Organização Mundial da Saúde. (2013). World suicide prevention day. Recuperado em fevereiro 4, 2013, de 
http://www.who.int/mediacentre/news/releases/2004/ pr61/es/index.html

Organização Mundial da Saúde. (2006). Prevenção do suicídio, um recurso para conselheiros. Genebra: Departamento de Saúde Mental e de Abuso de Substâncias. Recuperado em fevereiro 4, 2013, de http:// www.who.int/mental_health/media/counsellors_ portuguese.pdf

Pereira, A. (2011). Ideação suicida e fatores associados: estudo realizado numa amostra da população universitária da Universidade de Trás-os-Montes e Alto Douro (Tese de mestrado não-publicada). Universidade de Trás-os-Montes e Alto Douro, Vila Real, Portugal.

Prieto, D., \& Tavares, M. (2005). Fatores de risco para o suicídio e tentativa de suicídio: incidência, eventos estressores e transtornos mentais. Jornal Brasileiro de Psiquiatria, 54(2), 146-154.
Reynolds, W. (1988). Suicidal ideation questionnaire: Professional manual. Odessa: Psychological Assessment Resources.

Rivera, R. (2010). Suicidio y ansiedad. Revista Sinopsis, 46, 11-14.

Van Orden, K., Witte, T., Cukrowicz, K., Braithwaite, S., Selby, E., \& Joiner, T. (2010). The interpersonal theory of suicide. Psychological Review, 117(2), 575-600. http://dx.doi.org/10.1037/a0018697

Wilcox, H., Arria, A., Caldeira, K., Vincent, K., Pinchevsky, G., \& O'Grady, K. (2010). Prevalence and predictors of persistent suicide ideation, plans, and attempts during college. Journal of Affective Disorders, 127(1-3), 287-294. http://dx.doi.org/10.1016/j.jad.2010.04.017

Recebido: dezembro 11, 2013

Versão final: maio 28, 2015

Aprovado: julho 1, 2015 